\title{
Design of Composites, Catalysts, and Building Materials Using Insights from Simulation and Experiment
}

\author{
Hendrik Heinz \\ Department of Chemical and Biological Engineering, University of Colorado-Boulder \\ Boulder, CO 80309, USA \\ hendrik.heinz@colorado.edu
}

The development of functional materials continues to involve extensive trial-and-error studies while rational understanding and design using modeling and simulation play an increasing role due to more accurate models and affordable

computing resources. This talk describes multiscale simulation approaches to understand the prediction of properties of composites, catalysts, and building materials in correlation with experiment. At the nanoscale, a key role is played by recognition and assembly of different phases such as carbon nanotubes, polymer electrolytes, metals, and minerals. We will explain predictions of glass transition temperatures and interfacial shear strengths in CNT/PAN composites in all-atom resolution and their correlation with macroscale measurements. The second theme will be the interaction of metal nanostructures with organic compounds and the computational prediction of reaction rates in correspondence with experimental turnover frequency measurements. Third, grinding of cement minerals in ball mills and their interaction of organic compounds will be discussed using molecular dynamics at the nanoscale to discrete elements at the macroscale, which has guided the improvement of commercial products to reduce energy consumption. We will further discuss the underlying fundamental science of specific adsorption and assembly of (bio)macromolecules on metallic and oxide/hydroxide nanostructures, emphasizing the integration of simulations and measurements into an iterative feedback loop. In developing the Interface force field and a surface model database for compounds across the periodic table for simulations from the nanoscale to the microscale, we find that an accurate representation of chemical bonding, structures, and surface energies in models is essential. In addition, specific surface chemistry and electrolyte composition play a key role in correlating modeling and experiments, including reactive simulations towards chemical accuracy with inputs from quantum mechanics and experiment. 